\title{
Well-Posed Treatment of Space-Charge Layers in the Electroneutral Limit of Electrodiffusion
}

\author{
ADAM R. STINCHCOMBE \\ University of Michigan \\ YOICHIRO MORI \\ University of Minnesota \\ AND \\ CHARLES S. PESKIN \\ New York University
}

\begin{abstract}
The electroneutral model describes cellular electrical activity, accounting for ionic concentration dynamics without resolution of the fine spatial scales of the space-charge layer. This is done by asserting that the ionic solution is electrically neutral at each point in space. However, electroneutrality is inconsistent with the original boundary conditions at cell membranes. We consider three separate methods of resolving this inconsistency that result in well-posed models that are accurate approximations to a detailed model in which the space-charge layer is fully resolved. A particular electrodiffusion problem is utilized to make the discussion specific. (C) 2015 Wiley Periodicals, Inc.
\end{abstract}

\section{Introduction}

The electroneutral model was introduced by Mori et al. [15] as a detailed extension of the cable equation in electrophysiology. We shall present the model as developed previously [14, 15], beginning from the Poisson-Nernst-Planck (PNP) equations of electrodiffusion.

The concentrations $c_{i}(\boldsymbol{x}, t)$ of $N$ species of ions are tracked in regions of space separated by cell membranes. The ion concentrations are governed by the driftdiffusion equations:

$$
\begin{aligned}
\frac{\partial c_{i}}{\partial t} & =-\nabla \cdot \boldsymbol{f}_{i}, \\
\boldsymbol{f}_{i} & =-D_{i}\left(\nabla c_{i}+\frac{q z_{i} c_{i}}{k_{\mathrm{B}} T} \nabla \phi\right) .
\end{aligned}
$$

The flux of the $i^{\text {th }}$ species of ion is $\boldsymbol{f}_{i}$, which is expressed as the sum of a term from Fick's first law and a drift term. The diffusion constant of the $i^{\text {th }}$ ion species is $D_{i} ; q z_{i}$ is the amount of charge of one ion of the $i^{\text {th }}$ species, where $q$ is the 
elementary charge; $q D_{i} /\left(k_{\mathrm{B}} T\right)$ is the mobility of the $i^{\text {th }}$ ion species, where $k_{\mathrm{B}}$ is the Boltzmann constant and $T$ is the absolute temperature; and $\phi(x, t)$ is the electrostatic potential.

The electrostatic potential depends on the ion concentrations according to the Poisson equation,

$$
-\Delta \phi=\frac{1}{\epsilon}\left(\sum_{i=1}^{N} q z_{i} c_{i}+\rho_{0}\right),
$$

where $\rho_{0}(\boldsymbol{x})$ is the background charge that is permitted to vary in space but not in time, and $\epsilon$ is the (uniform) dielectric constant of the ionic solution. We shall refer to equations (1.1)-(1.3) as the PNP model. Rubinstein [18] provides an introduction to the topic of electrodiffusion, and Hyon et al. [6] discuss the energetics of PNP equations.

Biological membranes, which we idealize as infinitely thin (but with finite capacitance; see below), result in boundary conditions on the electrostatic potential as well as on the ionic concentrations. The potential and also the normal component of the electric displacement vector $\boldsymbol{D}=\epsilon \boldsymbol{E}$, where $\boldsymbol{E}$ is the electric field, should be continuous at the cell membrane. Thus, $\phi$ and $\epsilon \frac{\partial \phi}{\partial \boldsymbol{n}}$ should be continuous at the membrane. The unit normal vector to the membrane pointing from the side under consideration to the other side is $\boldsymbol{n}$. The boundary conditions on the ionic concentrations are

$$
q z_{i} \boldsymbol{f}_{i}(\boldsymbol{x}, t) \cdot \boldsymbol{n}(\boldsymbol{x})=j_{i}(\boldsymbol{x}, t),
$$

where $j_{i}$ is the transmembrane ion channel current per unit area contributed by the $i^{\text {th }}$ species of ion. The transmembrane currents will typically be a function of the position on the membrane, some gating variables, the potential difference across the membrane, and the concentrations of ions on each side of the membrane [15].

For the most part, the PNP model is intractable because of fine spatial and temporal scales, as discussed by Mori [13]. However, ionic solutions governed by the PNP model tend to be nearly electrically neutral away from membranes in parameter regimes of biological interest [15]. This fact is exploited in the electroneutral model, and we therefore assume that electroneutrality, expressed as

$$
\sum_{i=1}^{N} q z_{i} c_{i}+\rho_{0}=0
$$

holds at each point in space.

Within the PNP model, deviations from electroneutrality are only significant near membranes in what is known as the Debye layer. It has a length scale of the Debye length, defined as

$$
l_{\mathrm{D}}=\sqrt{\frac{\epsilon k_{\mathrm{B}} T}{q^{2} c_{0}}}
$$


where $c_{0}$ is a typical concentration of the ions near the membrane. This spacecharge layer arises because of an incompatibility between electroneutrality and the electrical boundary conditions.

In the electroneutral model, the Poisson equation, equation (1.3), is replaced by the electroneutrality condition, equation (1.4). This can be expressed as a constraint on $\phi$ by differentiating equation (1.4) with respect to time and substituting equation (1.1) and equation (1.2), which results in

$$
\nabla \cdot(a(\boldsymbol{x}, t) \nabla \phi+\nabla b(\boldsymbol{x}, t))=0,
$$

with

$$
a(\boldsymbol{x}, t)=\sum_{i=1}^{N} \frac{\left(q z_{i}\right)^{2} D_{i}}{k_{\mathrm{B}} T} c_{i}(\boldsymbol{x}, t), \quad b(\boldsymbol{x}, t)=\sum_{i=1}^{N} q z_{i} D_{i} c_{i}(\boldsymbol{x}, t) .
$$

The constraint on $\phi$, equation (1.6), is elliptic since $a(x, t)>0$ and can be interpreted as current continuity, $\nabla \cdot \boldsymbol{j}=0$, where $\boldsymbol{j}=-[a(\boldsymbol{x}, t) \nabla \phi+\nabla b(\boldsymbol{x}, t)]$ is the total electrical current density. Note that $\Delta \phi=0$ does not hold in general, which might incorrectly be expected from electroneutrality and equation (1.3).

The electrical boundary conditions of the PNP model are replaced by an analysis of the space-charge layer. The physics of the space-charge layer is approximated by considering there to be a surface charge density, $\sigma(x, t)$, on each side of the membrane. The cell membrane is assumed to have zero thickness and to be electrically neutral, which gives the relation $\sigma(\boldsymbol{x}, t)+\sigma^{\prime}(\boldsymbol{x}, t)=0$, where $\sigma^{\prime}(\boldsymbol{x}, t)$ is the surface charge density on the other side of the membrane. The surface charge density varies as a result of electrical currents at the membrane as well as transmembrane currents. Charge conservation at the membrane surface is expressed as

$$
\frac{\partial \sigma}{\partial t}(\boldsymbol{x}, t)=\boldsymbol{j}(\boldsymbol{x}, t) \cdot \boldsymbol{n}(\boldsymbol{x})-j_{\mathrm{m}}(\boldsymbol{x}, t)
$$

where $j_{\mathrm{m}}=\sum_{i=1}^{N} j_{i}$ is the transmembrane current per unit area from the side under consideration to the other side. Note that the transmembrane current is defined everywhere along the membrane, as the currents localized through ion channels have been averaged out. As before, $\boldsymbol{n}$ is the unit normal vector pointing from the side under consideration to the other side and $\boldsymbol{j}$ is the total electrical current density.

The membrane has a (constant) capacitance per unit area, $C_{\mathrm{m}}$, that relates the surface charge density to $\phi$ by $\sigma(\boldsymbol{x}, t)=C_{\mathrm{m}} \phi_{\mathrm{m}}(\boldsymbol{x}, t)$, where $\phi_{\mathrm{m}}(\boldsymbol{x}, t)=\phi(\boldsymbol{x}, t)-$ $\phi^{\prime}(\boldsymbol{x}, t)$ is the jump in potential across the membrane and its two space-charge layers. Unprimed quantities denote variables defined on the side of the membrane under consideration, while a prime will be used to denote quantities on the other side of the membrane. Note that by this definition the quantity $\phi_{\mathrm{m}}$ has equal magnitude but opposite sign on the two sides of the membrane. A schematic diagram of the model variables is given in Figure 1.1 . 


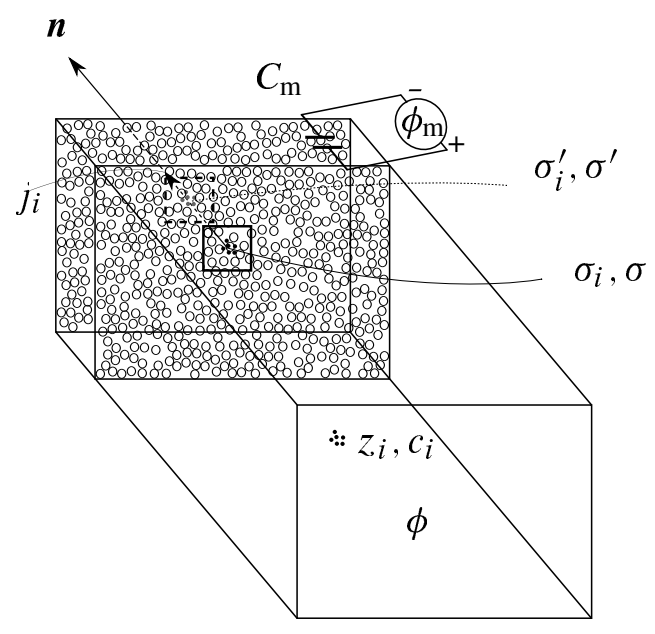

FiguRE 1.1. Definition of the variables in the electroneutral model. Everywhere within three dimensional space, there is an electrostatic potential, $\phi$, and for each ion species, with valance $z_{i}$, a concentration, $c_{i}$. On the membrane, there is a surface charge density for each ion, $\sigma_{i}$, and a total charge density, $\sigma$. They are functions of the location on the membrane. A prime denotes a quantity defined on the other side of the membrane. Each ion traverses the membrane with a current per unit area of $j_{i}$. This quantity is a function of the location on the membrane and should be viewed as a homogenization of ion channel currents. The unit normal vector $\boldsymbol{n}$ points from the side under consideration (unprimed variables) to the other side (with primed variables). The membrane is drawn with thickness for clarity, but within the model is idealized to have zero thickness. Despite having zero thickness, the membrane has a finite capacitance per unit area of $C_{\mathrm{m}}$. This is the total capacitance, including that from the bare membrane, $C$, and the two space-charge layers, which combine as capacitors in series, see equation 1.21. The potential difference across the membrane and its two space-charge layers is given as $\phi_{\mathrm{m}}(\boldsymbol{x}, t)=\phi(\boldsymbol{x}, t)-\phi^{\prime}(\boldsymbol{x}, t)$, where $\phi^{\prime}(\boldsymbol{x}, t)$ is the electrostatic potential on the other side of the membrane at position $x$ on the membrane.

In addition to total charge conservation, the number of ions of each individual species is conserved. Conservation at the membrane gives

$$
\frac{\partial \sigma_{i}}{\partial t}(\boldsymbol{x}, t)=q z_{i} \boldsymbol{f}_{i}(\boldsymbol{x}, t) \cdot \boldsymbol{n}(\boldsymbol{x})-j_{i}(\boldsymbol{x}, t) .
$$

$\sigma_{i}(\boldsymbol{x}, t)$ is the surface charge density of the $i^{\text {th }}$ ion species and therefore

$$
\sum_{i=1}^{N} \sigma_{i}(\boldsymbol{x}, t)=\sigma(\boldsymbol{x}, t) .
$$


Equation (1.7) results from summing over $i$ in equation (1.8).

We have introduced a surface charge density to resolve the inconsistency between the electroneutrality and the boundary conditions. An approximate analysis of the space-charge layer relates the surface charge density to the ion concentrations near the membrane [15]. Three assumptions are used:

(1) the space-charge layer is treated as one dimensional,

(2) all quantities in the space-charge layer are in thermodynamic equilibrium, and

(3) the deviations of ion concentrations and electrostatic potential from their bulk values are small.

The PNP model under these assumptions becomes

$$
\begin{aligned}
0 & =-D_{i}\left(\frac{d c_{i}}{d x}+\frac{q z_{i} c_{i}}{k_{\mathrm{B}} T} \frac{d \phi}{d x}\right), \\
-\frac{d \phi}{d x^{2}} & =\frac{1}{\epsilon}\left(\sum_{i=1}^{N} q z_{i} c_{i}+\rho_{0}\right) .
\end{aligned}
$$

The space variable, $x$, gives the distance from the membrane, along a line perpendicular to the membrane. Here $x=0$ is the location of the membrane and values of the bulk solution are obtained as $x \rightarrow \infty$. The bulk values $c_{i}(\infty)$ and $\phi(\infty)$ are considered to be known quantities, which is justified by the relatively slow time scales and long length scales over which the bulk values vary compared to the quantities in the space-charge layer. The background charge density, $\rho_{0}$, is effectively constant over the short length of the space-charge layer. The bulk solution is assumed to be electrically neutral: $\sum_{i=1}^{N} q z_{i} c_{i}(\infty)+\rho_{0}=0$.

Equation (1.9) is integrated to find

$$
c_{i}(x)=c_{i}(\infty) \exp \left(-\frac{q z_{i}}{k_{\mathrm{B}} T}(\phi(x)-\phi(\infty))\right) .
$$

This dependence of each ion concentration on the potential is substituted into equation (1.10), and the resulting second-order equation, known as the PoissonBoltzmann equation [4], is integrated once to give

$$
\begin{aligned}
\left(\frac{d \phi}{d x}\right)^{2}=\frac{2 k_{\mathrm{B}} T}{\epsilon} \sum_{i=1}^{N} c_{i}(\infty) & {\left[\exp \left(-\frac{q z_{i}}{k_{\mathrm{B}} T}(\phi(x)-\phi(\infty))\right)\right.} \\
& \left.-1+\frac{q z_{i}}{k_{\mathrm{B}} T}(\phi(x)-\phi(\infty))\right]
\end{aligned}
$$

The condition that $\frac{d \phi}{d x}(\infty)=0$ has been imposed. This first-order separable equation can be integrated again to give an implicit solution for $\phi(x)$ :

$$
x=\int_{\phi(0)}^{\phi} \frac{d \psi}{g(\psi-\phi(\infty))},
$$


where

$$
g(y)= \pm \sqrt{\frac{2 k_{\mathrm{B}} T}{\epsilon} \sum_{j=1}^{N} c_{j}(\infty)\left[\exp \left(-\frac{q z_{j}}{k_{\mathrm{B}} T} y\right)-1+\frac{q z_{j}}{k_{\mathrm{B}} T} y\right]}
$$

The + or - root in $g(y)$ is used when $\phi(0)<\phi(\infty)$ or $\phi(0)>\phi(\infty)$, respectively. From equation (1.12), $\phi(x)$ is monotonic and by equation (1.9), each of the $c_{i}(x)$ is monotonic. For negatively charged ions, $c_{i}(x)$ will increase or decrease as $\phi(x)$ does, and $c_{i}(x)$ will do the opposite for positively charged ions.

The reason for studying the space-charge layer is to determine the contribution $\sigma_{i}$ of each ion species to the total charge density on the membrane $\sigma$ in terms of the bulk variables. The amount of surface charge density, in excess of the bulk, of the $i^{\text {th }}$ ion species is

$$
\sigma_{i}=\int_{0}^{\infty} q z_{i}\left(c_{i}(x)-c_{i}(\infty)\right) d x
$$

The sign of each $\sigma_{i}$ will agree with the sign of $\phi(\infty)-\phi(0)$ regardless of the sign of $z_{i}$. The integral in equation (1.14) can be evaluated by substituting equation (1.11) into equation (1.14) and changing the variable of integration from $x$ to $\phi$. This gives

$$
\sigma_{i}=q z_{i} c_{i}(\infty) \int_{\phi(0)}^{\phi(\infty)} \frac{\exp \left(-\frac{q z_{i}}{k_{\mathrm{B}} T}(\phi-\phi(\infty))\right)-1}{g(\phi-\phi(\infty))} d \phi,
$$

where $g(y)$ is defined by equation (1.13).

An approximation will be used to simplify the above expression for $\sigma_{i}$. Note that the integrand in equation 1.15$)$ has a removable discontinuity at $\phi=\phi(\infty)$. The limiting value of the integrand at $\phi=\phi(\infty)$ is

$$
\sqrt{\frac{\epsilon}{k_{\mathrm{B}} T}} \frac{q z_{i}}{\sqrt{\sum_{j=1}^{N} q^{2} z_{j}^{2} c_{j}}} .
$$

After replacing the integrand of equation 1.15 with this constant, the expression for $\sigma_{i}$ becomes

$$
\sigma_{i} \approx \sqrt{\frac{\epsilon}{k_{\mathrm{B}} T}} \frac{q^{2} z_{i}^{2} c_{i}(\infty)}{\sqrt{\sum_{j=1}^{N} q^{2} z_{j}^{2} c_{j}(\infty)}}[\phi(\infty)-\phi(0)] .
$$

Summing over $i$ in equation 1.16 gives

$$
\sigma=\sum_{i=1}^{N} \sigma_{i} \approx \sqrt{\sum_{i=1}^{N} \frac{\epsilon q^{2} z_{i}^{2}}{k_{\mathrm{B}} T} c_{i}(\infty)}[\phi(\infty)-\phi(0)] .
$$


The contribution to the charge on the membrane from each ion can be expressed as a fraction of the total charge on the membrane as

$$
\sigma_{i} \approx \frac{z_{i}^{2} c_{i}(\infty)}{\sum_{j=1}^{N} z_{j}^{2} c_{j}(\infty)} \sigma .
$$

We have eliminated $\phi(0)$ in favor of bulk concentrations and $\sigma_{i}$ for which evolution equations have been specified above. Note that $\sigma$ is known in terms of bulk quantities since $\sigma=C_{\mathrm{m}} \phi_{\mathrm{m}}$, in which $C_{\mathrm{m}}$ is the total capacitance and $\phi_{\mathrm{m}}$ is the potential change across the membrane and its two space-charge layers. The total capacitance includes the bare capacitance of the membrane, $C$, and the charge separation associated with the two space-charge layers; see equation (1.21) below. In the notation of the analysis of the space-charge layers, $\phi_{\mathrm{m}}=\phi(+\infty)-\phi(-\infty)$.

Approximating the integrand in equation (1.15) as constant will be accurate provided $\phi(x)$ does not vary substantially within the space-charge layer. In particular, it must be true that

$$
\left|\frac{q z_{i}}{k_{\mathrm{B}} T}(\phi(x)-\phi(\infty))\right| \ll 1 .
$$

The same expression in equation (1.18) was obtained by Mori et al. [15] by linearizing before integrating the Poisson-Boltzmann equation. Here the approximation has occurred after integration of the Poisson-Boltzmann equation. An advantage of the approach followed here is that a more accurate treatment of the space-charge layer may be obtained by taking more terms in the Taylor series of the integrand about $\phi=\phi(\infty)$ in equation (1.15) or evaluating the integral in equation 1.15 numerically as required.

Note that $\phi(\infty)-\phi(0)$ is not the jump in $\phi$ across the membrane. It is the small deviation in the electric potential over the space-charge layer. Equation (1.17) shows that the space-charge layer acts as a capacitor, separating charge in proportion to the potential difference across it. Thus, we may assign a capacitance per unit area to the space-charge layer,

$$
C_{\mathrm{SCL}}=\sqrt{\sum_{i=1}^{N} \frac{\epsilon q^{2} z_{i}^{2}}{k_{\mathrm{B}} T} c_{i}(\infty)}
$$

and similiarly a capacitance per unit area to the space-charge layer on the other side of the membrane,

$$
C_{\mathrm{SCL}}^{\prime}=\sqrt{\sum_{i=1}^{N} \frac{\epsilon q^{2} z_{i}^{2}}{k_{\mathrm{B}} T} c_{i}^{\prime}(\infty)} .
$$

These two capacitors and the bare capacitance of the membrane per unit area, $C$, are in series, so the total membrane capacitance per unit area is given by

$$
\frac{1}{C_{\mathrm{m}}}=\frac{1}{C_{\mathrm{SCL}}}+\frac{1}{C}+\frac{1}{C_{\mathrm{SCL}}^{\prime}} .
$$


In the sum on the right-hand side of equation (1.21), the dominant term is $1 / C$, i.e., $C \ll C_{\mathrm{SCL}}$ and $C \ll C_{\mathrm{SCL}}^{\prime}$. Thus, $C_{\mathrm{m}} \approx C$.

The space-charge layer capacitance depends on the ionic concentrations near the membrane, as per equation (1.20). This suggests that the membrane capacitance should not be constant over the entire membrane. However, since the spacecharge layer capacitance contributes little to the membrane capacitance, the theory is consistent with the observations that the membrane capacitance does not depend on attributes of the space-charge layer unless the ionic concentrations are extreme [3, 19].

The space-charge layer analysis is applied locally to each patch of membrane. Thus,

$$
\sigma_{i}(\boldsymbol{x}, t)=\lambda_{i}(\boldsymbol{x}, t) \sigma(\boldsymbol{x}, t)
$$

where $\boldsymbol{x}$ is a location in space coincident with membrane and

$$
\lambda_{i}(\boldsymbol{x}, t)=\frac{z_{i}^{2} c_{i}(\boldsymbol{x}, t)}{\sum_{j=1}^{N} z_{j}^{2} c_{j}(\boldsymbol{x}, t)} .
$$

Our analysis of the space-charge layer thus gives the equations

$$
q z_{i} \boldsymbol{f}_{i}(\boldsymbol{x}, t) \cdot \boldsymbol{n}(\boldsymbol{x})=C_{\mathrm{m}} \frac{\partial}{\partial t}\left(\lambda_{i} \phi_{\mathrm{m}}\right)+j_{i}(\boldsymbol{x}, t),
$$

which provide boundary conditions for the evolution of the ion concentrations. These boundary conditions account for the effect of the space-charge layers without resolving them.

The electroneutral model is now fully specified. The ion concentrations $c_{i}(\boldsymbol{x}, t)$ satisfy equations $(1.1)-(1.2)$ with boundary conditions given by equation (1.24). The electric potential $\phi(\boldsymbol{x}, t)$ is subject to equation (1.6) with a jump across membranes given by $\phi_{\mathrm{m}}$, which evolves according to equation (1.24). The state variables of the model are (any) $N-1$ of the ion concentrations (the last concentration is determined by the electroneutral condition), the membrane potential, and any auxiliary variables, such as gating variables, needed to determine the transmembrane currents.

The electroneutral model is a good approximation of the PNP model in the limit as $l_{\mathrm{D}} \rightarrow 0$. A physiologically valid value of the Debye length is $l_{\mathrm{D}} \approx 1 \mathrm{~nm}$, which is small relative to the length scales within a neuron [13]. From equation (1.5), the limit $l_{\mathrm{D}} \rightarrow 0$ can be achieved by increasing the typical ion concentration, $c_{0} \rightarrow \infty$. As will be shown later, this is a self-consistent way to achieve $l_{\mathrm{D}} \rightarrow 0$ since the change in potential over the space-charge layer approaches 0 in this limit. According to equation (1.19), the approximation of the space-charge layer thus becomes more accurate in the limit as $c_{0} \rightarrow \infty$.

Unfortunately, as stated, the electroneutral model is ill-posed. Small perturbations to the ion concentrations near a membrane can grow exponentially in time. This phenomenon has been exhibited [13] in special cases of the model and an additional example will be presented here. 
In this paper, a modification of the electroneutral model that results in a wellposed model is presented. Additionally, it is shown how particular discretization of the original electroneutral model can be well-posed and in what sense the solutions of those discretized equations have meaning. A well-posed version of the electroneutral model previously presented [14] is also considered for comparison. We use the word stabilization to describe the transformation of an unstable, ill-posed problem into a stable, well-posed one. We avoid the use of the word regularization as that would incorrectly suggest additional smoothness assumptions.

\section{The Nature of the Instability and its Stabilization}

\subsection{The Finite Thickness Stabilization}

The source of the instability in the original electroneutral model will now be described. The excess number of ions of the $i^{\text {th }}$ species per unit area of membrane is given as $s_{i}=\frac{\sigma_{i}}{q z_{i}}=\frac{\lambda_{i} \sigma}{q z_{i}}=\frac{\lambda_{i} C_{\mathrm{m}} \phi_{\mathrm{m}}}{q z_{i}}$. Since, $\phi_{\mathrm{m}}$ has the same magnitude but opposite sign on the two sides of the membrane, the value of $s_{i}$ is negative on one side or the other of the membrane. The magnitude of $s_{i}$ is not the same on the two sides of the membrane since the concentrations of ions can be different on the two sides, but only its sign is currently relevant.

The negative values of $s_{i}$ are the source of the instability. This can be seen by investigating the effect of an increase in ion concentration when all other quantities are held fixed. To see how this change affects the excess ions on the membrane, consider the relative change in the excess number of ions:

$$
\frac{1}{s_{i}} \frac{\partial s_{i}}{\partial c_{i}}=\frac{1}{\lambda_{i}} \frac{\partial \lambda_{i}}{\partial c_{i}}=\frac{1}{\lambda_{i}} \frac{z_{i}^{2} \sum_{j=1, j \neq i}^{N} z_{j}^{2} c_{j}}{\left(\sum_{j=1}^{N} z_{j}^{2} c_{j}\right)^{2}}>0 .
$$

Since $\lambda_{i}=z_{i}^{2} c_{i} / \sum_{j=1}^{N} z_{j}^{2} c_{j}, \lambda_{i}$ is positive and is an increasing function of $c_{i}$. Thus, $s_{i}$ and $\frac{\partial s_{i}}{\partial c_{i}}$ have the same sign. For each ion, on the side of the membrane for which $s_{i}$ is negative, an increase in the concentration of an ion results in a decrease in the amount of that ion stored within the space-charge layer. Thus the increase in concentration near the membrane calls forth ions of the same type from the spacecharge layer causing a further increase in concentration. This is an unphysical positive feedback mechanism that amplifies small disturbances.

The trouble with the original formulation of the electroneutral model, then, is that only the deviations of concentration from the bulk values that occur within the space-charge layer have been considered. The bulk values themselves have not been included. This omission does not affect considerations of charge within the space-charge layer, since the bulk values are collectively electrically neutral, but it does affect the numbers of ions of each type that are stored there.

With regard to renormalization in quantum electrodynamics, Dirac is quoted as saying "Sensible mathematics involves neglecting a quantity when it is small—not 
neglecting it just because it is infinitely great and you do not want it!" [7]. In equation (1.14), the surface charges were considered to be only those in excess (or deficit) of that which would result from the bulk concentrations being extended into the space-charge layer. However, defining the surface charge density as

$$
\sigma_{i}=\int_{0}^{\infty} q z_{i} c_{i}(x) d x
$$

would not be useful as $q z_{i} c_{i}(x)$ is not integrable. In effect, an infinite contribution to $\sigma_{i}$ has been neglected in equation (1.14).

To account for the presence of ions at approximately their bulk concentrations not only away from the membrane but also near the membrane, a more physically reasonable definition of $s_{i}$ is

$$
s_{i}=l_{0} c_{i}(\infty)+\frac{\sigma_{i}}{q z_{i}},
$$

in which $l_{0}$ is a length scale greater than any Debye length in the electrodiffusion problem. The Debye length, $l_{\mathrm{D}}$, is defined in equation (1.5). Since the typical concentration of ions, $c_{0}$, varies along the membrane, so does $l_{\mathrm{D}}$. Select $l_{0}$ large enough so that it is larger than any Debye length present in the problem but smaller than any length scale of interest. The existence of such a length is a condition for the relevance of the electroneutral model. In the limit as $l_{\mathrm{D}} \rightarrow 0$, in which the electroneutral model is an accurate approximation of the PNP model, we shall take $l_{0} \rightarrow 0$, but in such a way that it is always larger than $l_{\mathrm{D}}$ to achieve the same agreement.

In equation (2.1), $\sigma_{i}$ remains defined as the excess surface charge as specified in equation (1.14). Since the bulk solution is electrically neutral, the first term in the new definition of $s_{i}$ makes no contribution to the total charge stored within the space-charge layer, provided that any background charge $\rho_{0}$ extends into the spacecharge layer, which we are assuming is the case. This new term is important when $c_{i}$ changes, since it contributes to the corresponding changes in $s_{i}$. In particular, the first term will dominate the second for all cases of interest and therefore $s_{i}$ and $\frac{\partial s_{i}}{\partial c_{i}}$ are always positive.

The claim that the first term in equation 2.1) dominates the second is essentially the same as the assumption that deviations of concentration from their bulk values that occur in the space-charge layer are small in comparison to the bulk values themselves throughout the space-charge layer. The condition that $\phi$ did not vary significantly in the space-charge layer, equation (1.19), was critical in the derivation of the expression of $\lambda_{i}$ in equation (1.23). Small changes in $\phi$ permit only small changes in the concentrations by equation (1.11). The restriction on the change in $\phi$ in the space-charge layer so that the first term in equation (2.1) dominates the second is not more restrictive than that needed for the validity of our previous analysis of the space-charge layer. We let $l_{0}=K l_{\mathrm{D}}$ where $K>1$ and $l_{\mathrm{D}}$ is the local Debye length. We impose $\left|\frac{\sigma_{i}}{q z_{i}}\right| \ll l_{0} c_{i}(\infty)$ and use the explicit 
expression for $\sigma_{i}$ in equation (1.16). This results in the condition

$$
\frac{1}{K}\left|\frac{q z_{i}}{k_{\mathrm{B}} T}(\phi(x)-\phi(\infty))\right| \ll \sqrt{\frac{\sum_{j=1}^{N} z_{j}^{2} c_{j}}{c_{0}}} .
$$

Any reasonable choice of $c_{0}$ would make the right-hand side of equation (2.2) order 1 or larger if there are many ion species. By comparing equation $(1.19)$ and equation (2.2), it is apparent that the new condition on the variation in $\phi$ over the space-charge layer is no more restrictive than that required for the space-charge layer analysis.

Conservation of ions at the membrane, originally expressed by equation (1.8), becomes

$$
\frac{\partial}{\partial t}\left(q z_{i} l_{0} c_{i}(\boldsymbol{x}, t)+\sigma_{i}(\boldsymbol{x}, t)\right)=q z_{i} \boldsymbol{f}_{i}(\boldsymbol{x}, t) \cdot \boldsymbol{n}(\boldsymbol{x})-j_{i}(\boldsymbol{x}, t) .
$$

The surface charge density, $\sigma_{i}$, is related to the bulk concentrations through the same space-charge layer approximation, equations (1.22)-(1.23). As this modification removes the instability of the original electroneutral model, we refer to it as the finite thickness stabilization. In the electroneutral model, the space-charge layer is approximated to have zero thickness, and its presence is accounted for by a boundary condition. In this stabilization, the space-charge layer is considered to have a finite thickness only for the purpose of counting the ions stored on the membrane surface.

\subsection{The Finite Volume Discretization Stabilization}

The details of a finite volume discretization of the electroneutral model are given by Mori and Peskin [16]. A useful observation is that a finite volume discretization of the original electroneutral model gives rise to a discretization error that acts exactly like the first term in equation 2.1). For a voxel that has membrane as part of its boundary, two variables are recorded for each species of ion in that voxel. One variable is the concentration $c_{i}$ and the other is the total number of ions $N_{i}$ in the voxel. The relationship between $N_{i}$ and $c_{i}$ is important, as $N_{i}$ is updated from the fluxes into the voxel, but we need $c_{i}$ to determine the fluxes. The correct relationship is $N_{i}=V c_{i}+N_{\mathrm{b}, i}$. The total volume of the voxel is $V$, including the volume taken up by the space-charge layer, and $N_{\mathrm{b}, i}$ is the excess number of ions of species $i$ in the space-charge layer. To be specific, $N_{\mathrm{b}, i}$ is computed in the original manner as $N_{\mathrm{b}, i}=A_{\mathrm{m}} \frac{\sigma_{i}}{q z_{i}}$, where $A_{\mathrm{m}}$ is the area of membrane facing the voxel.

The proposed modification to $s_{i}$ would add the term $A_{\mathrm{m}} l_{0} c_{i}$ to the calculation of $N_{i}$, but this is already included in the term $V c_{i}$ since $V$ includes the volume of the space-charge layer. With the new definition of $s_{i}$, the total number of ions in the voxel is given as $N_{i}=\left(V-l_{0} A_{\mathrm{m}}\right) c_{i}+A_{\mathrm{m}} s_{i}=V c_{i}+A_{\mathrm{m}} \frac{\sigma_{i}}{q z_{i}}$, which is the same as before. Small perturbations will not grow in the manner described since $\frac{\partial N_{i}}{\partial c_{i}}>0$. The instability will appear, as it should in a convergent discretization, 
faithfully reproducing the behavior in the continuous equations when the voxel size is so small that it fails to include the space-charge layer thickness. When the voxel size becomes smaller than the space-charge layer thickness, the first term in the expression for $N_{i}$ no longer dominates the second term. The claim here is that a finite volume discretization can stabilize the original electroneutral model in the same manner as the finite thickness stabilization described above.

\subsection{The Temporal Stabilization}

Another stabilization for the original electroneutral model that was previously presented [14] uses low-pass filtering of the boundary condition. The boundary condition will have a lagged influence by making each $\lambda_{i}$ a dynamic variable that tracks, with rate constant $\alpha$, the value it would have in the original electroneutral model.

\section{An Example of an Electrodiffusion Problem}

We consider a specific example of an electrodiffusion problem. Many essential properties of a more general electrodiffusion problem are retained, but the problem is kept simple enough that exact solutions are available to illustrate the approach of the solution of the electroneutral model to those of the PNP model. We shall demonstrate the instability of the original electroneutral model and show that the proposed stabilizations are successful. Both transient and steady-state agreement of the electroneutral and PNP model solutions will be shown explicitly.

\subsection{The PNP Model}

The domain considered will be three dimensional with $x, y$, and $z$ being the coordinate variables. A membrane will be located at $x=0$ and only the region $x>0$ will be considered. Two positive and monovalent ions have concentrations $p_{1}(x, y, z, t)$ and $p_{2}(x, y, z, t)$. A negative, monovalent ion has concentration $n(x, y, z, t)$. There are no background charges, $\rho_{0}=0$, and the ions have the same diffusion constants, $D=D_{1}=D_{2}=D_{3}$. The governing equations are

$$
\begin{aligned}
\frac{\partial p_{i}}{\partial t} & =D \Delta p_{i}+\frac{D q}{k_{\mathrm{B}} T} \nabla \cdot\left(p_{i} \nabla \phi\right), \quad i=1,2, \\
\frac{\partial n}{\partial t} & =D \Delta n-\frac{D q}{k_{\mathrm{B}} T} \nabla \cdot(n \nabla \phi), \\
-\Delta \phi & =\frac{q}{\epsilon}\left(p_{1}+p_{2}-n\right) .
\end{aligned}
$$

At the membrane $(x=0)$, the electric displacement field is continuous in the direction normal to the membrane,

$$
-C[\phi](y, z, t)=\epsilon \frac{\partial \phi}{\partial x}\left(0^{+}, y, z, t\right),
$$

where $[\phi](y, z, t)=\phi\left(0^{-}, y, z, t\right)-\phi\left(0^{+}, y, z, t\right)$ is the jump down in potential across the membrane. Because we are currently considering the PNP model, the 
space-charge layers are fully resolved and included within the domain. Thus, $C$ is the bare capacitance of the membrane and does not include any influence on the capacitance from the change in potential across the space-charge layers.

There is no ion flux through the membrane as we consider a membrane without ion channels for simplicity. This can be expressed as

$$
\begin{aligned}
\frac{\partial p_{i}}{\partial x}\left(0^{+}, y, z, t\right)+\frac{q}{k_{\mathrm{B}} T} p_{i} \frac{\partial \phi}{\partial x}\left(0^{+}, y, z, t\right) & =0, \quad i=1,2, \\
\frac{\partial n}{\partial x}\left(0^{+}, y, z, t\right)-\frac{q}{k_{\mathrm{B}} T} n \frac{\partial \phi}{\partial x}\left(0^{+}, y, z, t\right) & =0 .
\end{aligned}
$$

Infinitely far from the membrane we expect the ion concentrations to approach constants that give an electrically neutral solution, and we expect the electric potential also to approach a constant. This is expressed as $p \equiv p_{1}+p_{2} \rightarrow c_{*}, n \rightarrow c_{*}$, and $\phi \rightarrow-\phi_{*} / 2$ as $x \rightarrow \infty$. The constant that $\phi$ approaches is selected so that $\phi_{*}>0$ is the total jump down from $x=-\infty$ to $x=\infty$. This includes the jump down across the membrane and the two space-charge layers. The jump down across the membrane only is denoted by $[\phi]$.

This problem has an equilibrium solution:

$$
\begin{aligned}
& p=c_{*} \exp \left(-\frac{q}{k_{\mathrm{B}} T}\left(\phi+\frac{\phi_{*}}{2}\right)\right), \\
& n=c_{*} \exp \left(+\frac{q}{k_{\mathrm{B}} T}\left(\phi+\frac{\phi_{*}}{2}\right)\right), \\
& \phi=\frac{2 k_{\mathrm{B}} T}{q} \log \left(\frac{e^{x / l_{\mathrm{D}}}+A}{e^{x / l_{\mathrm{D}}}-A}\right)-\frac{\phi_{*}}{2},
\end{aligned}
$$

where $A=\tanh \left(\frac{q}{8 k_{\mathrm{B}} T}\left(\phi_{*}-[\phi]\right)\right)$ and $l_{\mathrm{D}}=\sqrt{\left(\epsilon k_{\mathrm{B}} T\right) /\left(2 c_{*} q^{2}\right)}$. Note that $l_{\mathrm{D}}$ is consistent with the Debye length in equation 1.5 with the typical concentration taken to be $2 c_{*}$, the total ion concentration at infinity. This solution can be confirmed to satisfy the governing equations, equations (3.1)- 3.3), the ion flux boundary conditions, equations (3.5)- (3.6), and the conditions at infinity stated above. Note that $\phi$ is independent of $y$ and $z$ and approaches $-\phi_{*} / 2$ on the spatial scale of the Debye length. The electrical boundary condition, equation (3.4), imposes a transcendental equation relating $[\phi]$ to $\phi_{*}$ :

$$
\sinh \left(\frac{q}{4 k_{\mathrm{B}} T}\left(\phi_{*}-[\phi]\right)\right)=\frac{q l_{\mathrm{D}} C}{2 \epsilon k_{\mathrm{B}} T}[\phi] .
$$

In equation (3.7), $\phi_{*}$ is treated as given and $[\phi]$ is unknown. There is always one solution for $[\phi] \in\left(0, \phi_{*} / 2\right)$. Since $\phi_{*}$ is independent of $y$ and $z$, equation (3.7) is solved once and $[\phi]$ is also independent of $y$ and $z$. Also, $\phi$ is independent of $y$ and $z$.

We consider transients of $\tilde{p}=p_{1}-p_{2}$ while $p=p_{1}+p_{2}, n$, and $\phi$ remain fixed in equilibrium. These transients have no effect on $p, n$, or $\phi$. Since $\phi$ is constant, 
the governing equation for $\tilde{p}$ is linear, which we shall analyze by separation of variables.

Make two definitions

$$
P(x, y, z)=\tilde{p}(x, y, z) \exp \left(\frac{q}{k_{\mathrm{B}} T}\left(\phi+\phi_{*} / 2\right)\right)
$$

and

$$
B(x)=\exp \left(-\frac{q}{k_{\mathrm{B}} T}\left(\phi+\phi_{*} / 2\right)\right)=\left(\frac{e^{x / l_{\mathrm{D}}}-A}{e^{x / l_{\mathrm{D}}}+A}\right)^{2},
$$

so that the governing equation and no-flux boundary conditions are

$$
\begin{gathered}
B(x) \frac{\partial P}{\partial t}=D \nabla \cdot(B(x) \nabla P), \\
\frac{\partial P}{\partial x}\left(0^{+}, y, z, t\right)=0, \\
\frac{\partial P}{\partial x}(\infty, y, z, t)=0 .
\end{gathered}
$$

We shall first show that all solutions to this problem are stable, which will be explicitly verified when analytical expressions for solutions are exhibited. Let

$$
E(t)=\int_{-\infty}^{\infty} \int_{-\infty}^{\infty} \int_{0}^{\infty} B(x) \frac{P^{2}}{2} d x d y d z
$$

We find that $E(t)$ is a nonincreasing function of time since

$$
\begin{aligned}
\frac{d E}{d t} & =\int_{-\infty}^{\infty} \int_{-\infty}^{\infty} \int_{0}^{\infty} B(x) \frac{\partial}{\partial t}\left(\frac{P^{2}}{2}\right) d x d y d z \\
& =\int_{-\infty}^{\infty} \int_{-\infty}^{\infty} \int_{0}^{\infty} P D \nabla \cdot(B(x) \nabla P) d x d y d z \\
& =-D \int_{-\infty}^{\infty} \int_{-\infty}^{\infty} \int_{0}^{\infty} B(x)|\nabla P|^{2} d x d y d z \\
& \leq 0
\end{aligned}
$$

which follows from $B(x)>0$, an integration by parts, and equations (3.8)-3.10). Equality occurs only if $P$ is constant. Thus $E(t) \leq E(0)$ and solutions cannot grow in time. Since $E$ is a positive definite functional of $P$, all solutions of equations 3.8 -3.10) approach a constant solution.

By separating variables, exact solutions for $P$ can be obtained of the form

$$
P=X(x) \sin (\gamma y+\delta z) e^{\beta t},
$$

where

$$
\beta / D+\gamma^{2}+\delta^{2}=-\eta
$$


and $X(x)$ satisfies a linear ordinary differential equation,

$$
-X^{\prime \prime}-\frac{B^{\prime}}{B} X^{\prime}=\eta X
$$

and a boundary condition,

$$
X^{\prime}(0)=0 .
$$

The expression for the variable coefficient in the differential equation can be simplified to

$$
\frac{B^{\prime}}{B}=\frac{4}{l_{\mathrm{D}}}\left(\frac{1}{A} e^{x / l_{\mathrm{D}}}-A e^{-x / l_{\mathrm{D}}}\right)^{-1} .
$$

The no-flux condition at infinity, equation (3.10), will be imposed after synthesis of the eigenfunctions, but not on the individual eigenfunctions. So that this is possible, we require that the individual eigenfunctions remain bounded as $x \rightarrow \infty$. The form for $P$ in equation (3.12) does not give a finite energy from equation (3.11). Solutions will have finite energy after synthesis.

The condition that the eigenfunctions must be bounded implies that the eigenvalues $\eta$ are real and nonnegative as follows. Let $\eta=-\mu^{2}$ with $\mu$ complex. We will consistently denote the separation constant as $\eta$ and express it in terms of $\mu$, but we will change that expression and whether $\mu$ is complex or real to suit the current discussion. We hope that this is an excusable abuse of notation.

A closed-form expression for the eigenfunctions $X(x)$ is

$$
\begin{aligned}
X(x)=\frac{1}{1-A e^{-x / l_{\mathrm{D}}}}\{ & \left(2 \mu l_{\mathrm{D}}-1\right)\left[\left(2 \mu l_{\mathrm{D}}-1\right) A-\left(2 \mu l_{\mathrm{D}}+1\right)\right] e^{\mu x} \\
& +\left(2 \mu l_{\mathrm{D}}+1\right)\left[\left(2 \mu l_{\mathrm{D}}+1\right) A-\left(2 \mu l_{\mathrm{D}}-1\right)\right] e^{-\mu x} \\
& +A\left(2 \mu l_{\mathrm{D}}+1\right)\left[\left(2 \mu l_{\mathrm{D}}-1\right) A-\left(2 \mu l_{\mathrm{D}}+1\right)\right] e^{\left(\mu-1 / l_{\mathrm{D}}\right) x} \\
& \left.+A\left(2 \mu l_{\mathrm{D}}-1\right)\left[\left(2 \mu l_{\mathrm{D}}+1\right) A-\left(2 \mu l_{\mathrm{D}}-1\right)\right] e^{-\left(\mu+1 / l_{\mathrm{D}}\right) x}\right\} .
\end{aligned}
$$

Thus, the eigenfunctions are bounded if and only if $\operatorname{Re}(\mu)=0$. When $\operatorname{Re}(\mu)=0$, $\eta$ is real and nonnegative.

If the eigenvalue is $0, \eta=0$, then the only eigenfunctions satisfying $X^{\prime}(0)=0$ and remaining bounded as $x \rightarrow \infty$ are constant in $x$.

If the eigenvalue is positive, $\eta=\mu^{2}$ ( $\mu$ is real and without loss of generality take $\mu>0$ ), then a closed-form expression for the eigenfunctions is

$$
\begin{aligned}
X(x)= & 4 A \mu l_{\mathrm{D}} \frac{e^{x / l_{\mathrm{D}}}+1}{e^{x / l_{\mathrm{D}}}-A} \sin (\mu x) \\
& +\left[1+A+\left(2 \mu l_{\mathrm{D}}\right)^{2}(1-A) \frac{e^{x / l_{\mathrm{D}}}+A}{e^{x / l_{\mathrm{D}}}-A}\right] \cos (\mu x) .
\end{aligned}
$$

Every value of $\mu$ gives a bounded, nonzero eigenfunction and $\beta$ is determined by $\mu$. Indeed, since $\beta=-D\left(\gamma^{2}+\delta^{2}+\mu^{2}\right)$, we have $\beta<0$ and the solutions are 
stable. For large $x$, the eigenfunctions are sinusoidal,

$$
X(x) \sim 4 A \mu l_{\mathrm{D}} \sin (\mu x)+\left[1+A+\left(2 \mu l_{\mathrm{D}}\right)^{2}(1-A)\right] \cos (\mu x) .
$$

Having obtained explicit solutions for $\tilde{p}=p_{1}-p_{2}$ and shown their stability in the PNP model, we consider the electroneutral model and the proposed stabilizations.

\subsection{The Electroneutral Model and Stabilizations}

We shall now formulate the previously described electrodiffusion problem using the electroneutral model. To be consistent with the above, $\phi$ is piecewise constant with value $-\phi_{*} / 2$ for $x>0$ and $\phi_{*} / 2$ for $x<0$. In the electroneutral limit, $n=p=c_{*}$. The positive ion concentrations $p_{i}$ satisfy the diffusion equation and there is no ion flux at infinity:

$$
\frac{\partial p_{i}}{\partial t}=D \Delta p_{i}, \quad 0=-D \frac{\partial p_{i}}{\partial x}(\infty, y, z, t) .
$$

At the membrane, by equation 1.22 and equation 1.23 ,

$$
L \frac{\partial p_{i}}{\partial t}\left(0^{+}, y, z, t\right)=D \frac{\partial p_{i}}{\partial x}\left(0^{+}, y, z, t\right)
$$

where $L=-\frac{C_{\mathrm{m}} \phi_{*}}{2 q c_{*}}$.

We look for solutions for $\tilde{p}=p_{1}-p_{2}$ of the form

$$
\tilde{p}(x, y, z, t)=X(x) \sin (\gamma y+\delta z) e^{\beta t} .
$$

This ansatz requires

$$
\beta / D+\gamma^{2}+\delta^{2}=-\eta,
$$

and $X(x)$ satisfies a linear differential equation,

$$
-X^{\prime \prime}=\eta X
$$

The boundary condition requires

$$
L \beta X(0)=D X^{\prime}(0) .
$$

Let $\eta=-\mu^{2}$ with $\mu$ complex. A closed-form expression for the eigenfunctions is

$$
X(x)=\left[\mu+L\left(\mu^{2}-\gamma^{2}-\delta^{2}\right)\right] e^{\mu x}+\left[\mu-L\left(\mu^{2}-\gamma^{2}-\delta^{2}\right)\right] e^{-\mu x} .
$$

The eigenfunctions are bounded if $\operatorname{Re}(\mu)=0$. If $\operatorname{Re}(\mu)>0$ (or $\operatorname{Re}(\mu)<0$ ), so that the eigenfunctions are bounded, $\mu$ must satisfy $\mu+L\left(\mu^{2}-\gamma^{2}-\delta^{2}\right)=0$ (or $\mu-L\left(\mu^{2}-\gamma^{2}-\delta^{2}\right)=0$ ), which always has two real roots for $\mu$. Thus, $\eta$ is real and we separately consider it to be zero, negative, or positive.

For an eigenvalue of zero, $\eta=0$, there are no bounded, nonzero eigenfunctions.

For negative eigenvalues, $\eta=-\mu^{2}$ ( $\mu$ is real and without loss of generality take $\mu>0$ ), the eigenfunctions are

$$
X(x)=e^{-\mu x}
$$


with $\mu$ the positive root of

$$
L \mu^{2}+\mu-L\left(\gamma^{2}+\delta^{2}\right)=0 .
$$

There is one eigenfunction for each $\mu>0$. From equation 3.17, $\beta=-\frac{D \mu}{L}$ and therefore when $L>0$ this solution is stable, and when $L<0$ this solution is unstable for all frequencies $\gamma, \delta$.

For positive eigenvalues, $\eta=\mu^{2}$ ( $\mu$ is real and without loss of generality take $\mu>0$ ), the eigenfunctions are

$$
X(x)=\cos (\mu x+\theta)
$$

with $\mu$ being either of the positive roots of

$$
L \mu^{2}-\mu \tan \theta+L\left(\gamma^{2}+\delta^{2}\right)=0 .
$$

These roots are

$$
\mu=\frac{\tan \theta}{2 L} \pm \sqrt{\left(\frac{\tan \theta}{2 L}\right)^{2}-\left(\gamma^{2}+\delta^{2}\right)} .
$$

Both roots are positive if $L>0$ and $\theta \in\left(\theta_{*}, \pi / 2\right)$ or if $L<0$ and $\theta \in$ $\left(-\pi / 2,-\theta_{*}\right)$, where $\theta_{*}=\arctan \left(2|L| \sqrt{\gamma^{2}+\delta^{2}}\right)$. Equation (3.20) gives $\mu$ as two functions of $\theta$. The union of the ranges of these two functions is $(0, \infty)$ so that any positive $\mu$ corresponds to one eigenfunction by selecting the appropriate value of $\theta$. From equation (3.16), $\beta<0$ and these positive eigenvalue solutions are stable for all frequencies $\gamma$ and $\delta$.

The stable, positive-eigenvalue eigenfunctions are faithful representations of the PNP model in the limit as $c_{*} \rightarrow \infty$. For $l_{\mathrm{D}} \rightarrow 0$ by $c_{*} \rightarrow \infty$, from equation (3.7), $[\phi] \rightarrow \phi_{*}$ so that the changes in potential over the space-charge layers are negligible. According to equation (1.19), the approximation of the space-charge layer becomes more accurate in the limit $c_{*} \rightarrow \infty$. Another consistent way to achieve $l_{\mathrm{D}} \rightarrow 0$ is $T \rightarrow 0$, but not $\epsilon \rightarrow 0$. In the latter limit, from equation 3.7, $[\phi] \rightarrow 0$, which is inconsistent with the condition in equation (1.19).

Small $l_{\mathrm{D}}$ ensures that the electroneutral model eigenfunctions are accurate approximations to the PNP model eigenfunctions. From equation (3.14), the PNP model eigenfunctions become proportional to $\cos (\mu x)$ in the zero $l_{\mathrm{D}}$ limit since $A \rightarrow 0$ as $[\phi] \rightarrow \phi_{\star}$. In the electroneutral model as $c_{*} \rightarrow \infty, L \rightarrow 0$ and $\theta_{*} \rightarrow 0$. When $L \rightarrow 0$, we must take $\theta \rightarrow 0$ or else the two roots to equation (3.19) approach 0 and infinity. We take $L \rightarrow 0$ and $\theta \rightarrow 0$ so that $\tan \theta / L$ is fixed. In this limit, any value of $\mu$ corresponds to an eigenfunction as long as the fixed value of $\tan \theta / L$ is selected appropriately. Since $\theta \rightarrow 0$, the electroneutral model gives the same eigenfunctions as the PNP model. Equations 3.13 and 3.16 are the same, so the temporal decay rate, $\beta$, is the same for the two models for each eigenfunction.

The negative-eigenvalue eigenfunction either becomes constant or disappears depending on the sign of $L$ in the limit as $c_{*} \rightarrow \infty$. As $L \rightarrow 0^{+}$, from equation 
(3.18) the positive root for $\mu \rightarrow 0, \beta \rightarrow-D\left(\gamma^{2}+\delta^{2}\right)$, and $X(x)$ approaches a constant. This eigenfunction becomes the constant-in- $x$ solution of the PNP model. As $L \rightarrow 0^{-}$, the positive root for $\mu \rightarrow+\infty, \beta \rightarrow+\infty$, and $X(x)$ approaches 0 . When $L$ is negative, the unstable eigenfunction of the electroneutral model disappears in the limit $c_{*} \rightarrow \infty$. However, for finite $c_{*}$, this unstable eigenfunction is present. Since the temporal growth rate $\beta$ is unbounded, it can be made arbitrarily large by choosing $\gamma$ or $\delta$ sufficiently large; we see that the original electroneutral model is ill-posed.

The electroneutral model agrees with the PNP model in the limit as $c_{*} \rightarrow \infty$. Both models vary as $c_{*}$ is increased and their respective eigenfunctions are the same in the limit.

The electroneutral model is ill-posed when $L<0$. However, all is not well when $L>0$. Here, we considered the $x>0$ side of the membrane. From the $x<0$ perspective, $L^{\prime}=-L$ since $\phi_{*}^{\prime}=-\phi_{*}$ and $L \phi_{*}<0$. Thus, if $L>0$ and the $x>0$ side of the membrane is well-posed, then the $x<0$ side is ill-posed.

The ill-posedness of the electroneutral model has now been illustrated explicitly on this electrodiffusion problem. We shall now demonstrate how our proposed stabilizations alleviate the instability.

\section{The Finite Thickness Stabilization}

Applying the finite thickness stabilization, we replace the boundary condition in equation 3.15 by

$$
\widetilde{L} \frac{\partial p_{i}}{\partial t}\left(0^{+}, y, z, t\right)=D \frac{\partial p_{i}}{\partial x}\left(0^{+}, y, z, t\right),
$$

in which $\tilde{L}=l_{0}-\frac{C_{\mathrm{m}} \phi_{*}}{2 q c_{*}}$. Since $l_{0}$ dominates $\frac{C_{\mathrm{m}} \phi_{*}}{2 q c_{*}}, \tilde{L}>0$. On the $x<0$ side of the membrane, $\widetilde{L}^{\prime}=l_{0}+\frac{C_{\mathrm{m}} \phi_{*}}{2 q c_{*}}>0$. According to the analysis of the previous section, the electroneutral model with the finite thickness stabilization is well-posed for this example on both sides of the membrane.

Moreover, the transient solutions agree with those of the PNP model as $c_{*} \rightarrow$ $\infty$. The Debye length is uniform over the membrane and as $c_{*} \rightarrow \infty, l_{\mathrm{D}} \rightarrow 0$. We take $l_{0} \rightarrow 0$ and therefore $\tilde{L} \rightarrow 0$. As detailed previously, as $L \rightarrow 0^{+}$, the eigenfunctions of the electroneutral model agree with those of the PNP model. For this electrodiffusion problem, the finite thickness stabilization of the electroneutral model agrees with the PNP model as $c_{*} \rightarrow \infty$ and is well-posed for finite $c_{*}$.

\section{The Finite Volume Discretization Stabilization}

For simplicity, we discretize the $x$ dimension only and let $\tilde{p}_{k}(y, z, t)$ be an approximation to $\tilde{p}\left(\left(k-\frac{1}{2}\right) \Delta x, y, z, t\right)$ for $k=1,2, \ldots$. We keep the domain periodic in the $y$-and $z$-directions. The semidiscrete equations are

$$
\Delta x \frac{\partial \tilde{p}_{k}}{\partial t}=\Delta x D\left(\frac{\partial^{2} \tilde{p}_{k}}{\partial y^{2}}+\frac{\partial^{2} \tilde{p}_{k}}{\partial z^{2}}\right)+D \frac{\tilde{p}_{k+1}-\tilde{p}_{k}}{\Delta x}-D \frac{\tilde{p}_{k}-\tilde{p}_{k-1}}{\Delta x}
$$


for $k=2,3, \ldots$ Equation (3.21) can be rewritten as

$$
\frac{\partial \tilde{p}_{k}}{\partial t}=D\left(\frac{\partial^{2} \tilde{p}_{k}}{\partial y^{2}}+\frac{\partial^{2} \tilde{p}_{k}}{\partial z^{2}}+\frac{\tilde{p}_{k+1}-2 \tilde{p}_{k}+\tilde{p}_{k-1}}{(\Delta x)^{2}}\right) .
$$

The first voxel, $k=1$, is special since there is no flux from the left and also because of the storage of ions on the membrane at $x=0$. These stored ions are included within the first voxel. A discretization we shall use for this voxel is

$$
(L+\Delta x) \frac{\partial \tilde{p}_{1}}{\partial t}=\Delta x D\left(\frac{\partial^{2} \tilde{p}_{1}}{\partial y^{2}}+\frac{\partial^{2} \tilde{p}_{1}}{\partial z^{2}}\right)+D \frac{\tilde{p}_{2}-\tilde{p}_{1}}{\Delta x},
$$

which can be rewritten as

$$
\frac{\partial \tilde{p}_{1}}{\partial t}=\frac{\Delta x D}{L+\Delta x}\left(\frac{\partial^{2} \tilde{p}_{1}}{\partial y^{2}}+\frac{\partial^{2} \tilde{p}_{1}}{\partial z^{2}}+\frac{\tilde{p}_{2}-\tilde{p}_{1}}{(\Delta x)^{2}}\right) .
$$

We search for solutions to our semidiscretized problem of the form $\tilde{p}_{k}(y, z, t)=$ $X_{k} \sin (\gamma y+\delta z) e^{\beta t}$ for $k=1,2, \ldots$. The sequence $X_{k}$ may be complex valued, in which case the real part of $\tilde{p}_{k}$ is the desired solution. Equation (3.22) is satisfied if and only if

$$
\frac{\beta}{D}+\gamma^{2}+\delta^{2}=-\eta=\frac{1}{(\Delta x)^{2}} \frac{X_{k+1}-2 X_{k}+X_{k-1}}{X_{k}} .
$$

The linear difference equation for $X_{k}$ has a general solution that is a linear combination of solutions of the form $X_{k}=w^{k}$ with $w$ given by

$$
w_{ \pm}=1-\frac{1}{2} \eta(\Delta x)^{2} \mp \frac{1}{2} \Delta x \sqrt{\eta} \sqrt{\eta(\Delta x)^{2}-4} .
$$

We require that $X_{k}$ remain bounded. If $\eta$ is real and $0 \leq \eta(\Delta x)^{2} \leq 4$, then $w_{ \pm}$are complex and $\left|w_{ \pm}\right|=1$. When $\eta$ is not real or $\eta$ is real and $0>\eta$ or $\eta(\Delta x)^{2}>4$, we find that $\left|w_{-}\right|<1$ and $\left|w_{+}\right|>1$. Thus, in the latter case only one of the two roots contributes to the general, bounded solution, which we consider first. The former case is considered when we take a limit to recover the PNP model solutions.

Let $X_{k}=w^{k}$ and apply the boundary condition, equation 3.23 , to get

$$
\frac{\beta}{D}=-\frac{\Delta x}{L+\Delta x}\left(\gamma^{2}+\delta^{2}\right)+\frac{w-1}{\Delta x(L+\Delta x)} .
$$

Since $|w|<1$ implies $\operatorname{Re}(w-1)<0$, by equation $(3.26)$ if $L+\Delta x>0$ then $\operatorname{Re}(\beta)<0$. Similarly, $L+\Delta x<0$ results in instability, $\operatorname{Re}(\beta)>0$. Ignoring the stability of the boundary case, $L+\Delta x>0$ is equivalent to stability provided there exists a solution to equation (3.24) and equation (3.26) with $|w|<1$. We shall provide explicit expressions for these solutions.

Combining equation (3.24) with $X_{k}=w^{k}$ and equation (3.26) gives a quadratic equation for $w$,

$$
w^{2}-\left[\left(1+\frac{\Delta x}{L}\right)+\left(1+(\Delta x)^{2}\left(\gamma^{2}+\delta^{2}\right)\right)\right] w+\left(1+\frac{\Delta x}{L}\right)=0,
$$


with solutions

$$
w_{ \pm}=1+\frac{\chi+\chi^{2} \omega^{2}}{2} \pm \frac{1}{2} \sqrt{\left(\chi+\chi^{2} \omega^{2}\right)^{2}+4 \chi^{2} \omega^{2}}
$$

in which $\chi=\frac{\Delta x}{L}$ is the dimensionless voxel size and $\omega=|L| \sqrt{\gamma^{2}+\delta^{2}}$ is a dimensionless frequency. Note that $w_{+} w_{-}=1+\chi$ and $w_{+}+w_{-}=2+\chi+\chi^{2} \omega^{2}$. Also, $\omega>0$, but $\chi \in \mathbb{R}$ since $L$ may take on both positive and negative values. The boundary condition has been used to remove $\eta$ from the expression for the roots in equation (3.25). The roots given in equation (3.28) are always real and hence $\eta$ and $\beta$ appearing in equation (3.24) are real. Consideration of equation (3.28) leads to the following conclusions:

(1) $\left|w_{+}\right|>1$;

(2) if $\chi>-1$, then $0<w_{-}<1$;

(3) if $-2<\chi<-1$, then $-1<w_{-}<0$; and

(4) if $\chi<-2$, then $\chi^{2} \omega^{2}>-2(\chi+2)$ must hold so that $-1<w_{-}<0$.

The above results are summarized in Figure 3.1. In the shaded regions, there is a solution to equation (3.27) satisfying $|w|<1$. This solution is always $w_{-}$. For $L>0, \chi>0$ and there is a stable solution for all $\Delta x$. For $L<0$, there is a stable solution for all $\omega$ for $-L<\Delta x<-2 L$. If $\Delta x$ is reduced so that $\Delta x<-L$, then there exists a solution, but it is unstable. Additionally, there is a stable solution for all $\Delta x<-L$ as long as $\omega>\frac{1}{2}$. For low frequencies, $\omega<\frac{1}{2}$, a stable solution exists for $\Delta x$ in the range $-L<\Delta x<\left(1-\sqrt{1-4 \omega^{2}}\right) / \omega^{2}(-L)$.

For positive $L$, the finite volume discretization stabilization gives a stable solution for every frequency. For negative $L$, for which the original electroneutral model is unstable for all frequencies, the finite volume discretization stabilization is stable for all frequencies provided $-L<\Delta x$. If $\Delta x$ is reduced below $-L$, then the instability of the continuous equations of the original electroneutral model is reproduced. Thus, the finite volume stabilization is effective provided the voxel size is not too small. This makes intuitive sense as the original electroneutral model is not intended to resolve such fine scales. When $\Delta x<-L$, the width of the voxels is not long enough to include the length $|L|$. Since the surface charge is no longer included in the voxels beside the membrane, we do not expect stability.

To recover the PNP model solutions, we take $L \rightarrow 0$ and $\Delta x \rightarrow 0$ so that $\chi$ is fixed. From equation (3.28), $w_{-} \rightarrow 1+\chi$ if $\chi<0$ and $w_{-} \rightarrow 1$ if $\chi>0$. When $\chi>0$, the discrete solution approaches the constant in $x$ solutions of the PNP model. For stable choices of $\chi<0,1+\chi$ is between -1 and 0 and the limiting discrete solutions are identically zero.

To recover the positive eigenvalue solutions of the PNP model, take $X_{k}=w_{-}+$ $\tau w_{+}$with $w_{ \pm}$given by equation 3.25 when $0<\eta(\Delta x)^{2}<4$. These solutions are necessarily stable since $\eta>0$ implies $\beta<0$ from equation (3.24). The constant $\tau$ 


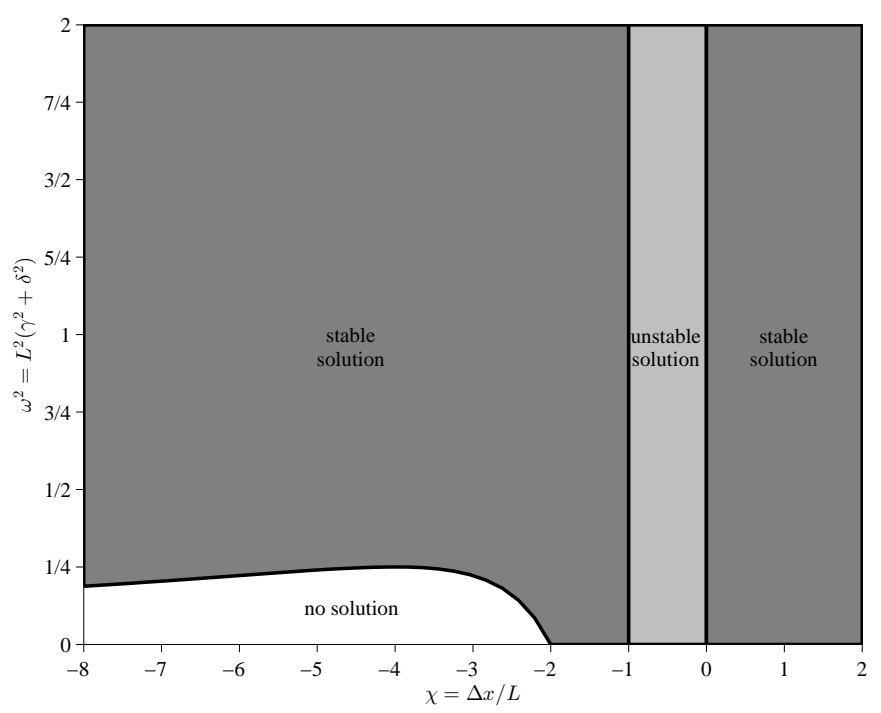

FIGURE 3.1. Regions in which a solution to equation (3.27) satisfies $|w|<1$ with $\chi=\frac{\Delta x}{L}$ and $\omega^{2}=L^{2}\left(\gamma^{2}+\delta^{2}\right)$. The region without a solution is separated from the region with a solution by the curve $w^{2}=$ $\frac{-2(\chi+2)}{\chi^{2}}$ with a maximum at $\chi=-4, \omega^{2}=\frac{1}{4}$. A stable solution, with $\operatorname{Re}(\beta)<0$, results for $\chi<-1$ or $\chi>0$. For $-1<\chi<0$, the solution is unstable. For $L>0, \chi>0$ and there is a stable solution for all $\Delta x$. For $L<0$, there is a stable solution for all $\omega$ for $-L<\Delta x<-2 L$. If $\Delta x$ is reduced so that $\Delta x<-L$, then there exists a solution, but it is unstable. Additionally, there is a stable solution for all $\Delta x<-L$ as long as $\omega>\frac{1}{2}$. For low frequencies, $\omega<\frac{1}{2}$, a stable solution exists for $\Delta x$ in the range $-L<\Delta x<\frac{-L}{\omega^{2}}\left(1-\sqrt{1-4 \omega^{2}}\right)$. In the no-solution region, the semidiscrete equations have a stable solution with $X_{k}=w_{-}+\tau w_{+}$ where $w_{ \pm}$is given by equation 3.25) with $0<\eta(\Delta x)^{2}<4$.

can be determined by applying the boundary condition, equation (3.23), to get

$$
\tau=-\frac{w_{-}}{w_{+}} \frac{\eta(\Delta x)^{2}(\chi+1)+\omega^{2} \chi^{2}+\chi\left(w_{-}-1\right)}{\eta(\Delta x)^{2}(\chi+1)+\omega^{2} \chi^{2}+\chi\left(w_{+}-1\right)} .
$$

In the limit $\Delta x \rightarrow 0$ and $\omega \rightarrow 0$ with $\eta$ and $\chi$ fixed, $X_{k}$ approaches a uniform sampling of $2 \cos (\sqrt{\eta} x)$. This approach is shown in Figure 3.2. Note these solutions become valid for any $\eta>0$ for sufficiently small $\Delta x$. Thus, for this electrodiffusion problem, the finite volume discretization stabilization gives stable solutions that are good approximations to those of the PNP model.

The finite volume discretization stabilization has the advantage over the finite thickness stabilization in that it does not explicitly use the Debye length. In the finite thickness stabilization, one must choose a typical concentration in order to obtain a value for the Debye length from equation (1.5) and completely specify 


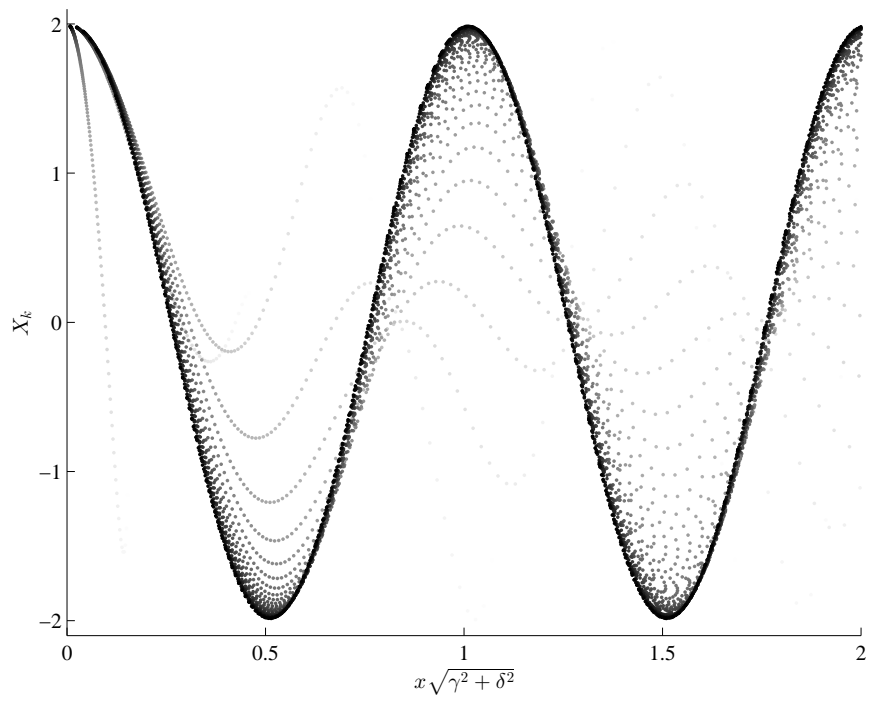

FIgURE 3.2. The solution $X_{k}$ as $\Delta x \rightarrow 0$ (and $L \rightarrow 0$ ) with $\chi=-\frac{3}{2}$ and $\eta=(2 \pi)^{2}\left(\gamma^{2}+\delta^{2}\right)$ fixed. Values of $\Delta x$ between 0 and $\frac{2}{\sqrt{\eta}}$, a total of 150, are shown with different grayscale colors (dark is small $\Delta x$ ). The values of $X_{k}$ approach a uniform sampling of $2 \cos (\sqrt{\eta} x)$.

the model. This is not necessary in the finite volume discretization stabilization. However, it suffers from a lower limit on the amount of grid refinement permitted. Also, the parameter determining the grid size is a free parameter analogous to the typical concentration in the finite thickness stabilization.

In order to use the finite volume discretization stabilization, the traditional concept of a model of a physical phenomenon must be modified. Typically, a model is expressed as a partial differential equation and appropriate boundary conditions. In the absence of analytical solutions, the continuous equations are discretized and solved on a computer to produce accurate approximations to the solutions of the model. More refinement gives more accurate approximations, but the model remains encapsulated in the continuous equations. Since the original electroneutral model is unstable, it cannot be a reasonable model of a physical phenomenon. In the electroneutral model with the finite volume discretization, the discrete equations are the model. The amount of refinement determines the accuracy of the model itself and not the accuracy of approximations to the model. By design, the solutions to the discrete equations will be similar to solutions of the PNP model when the assumption of electroneutrality is valid.

This perspective is not unique to our work, nor even to modeling cellular electrical activity. A similar approach in which circuit approximations are made to an electrical model has been used in modeling the T-tubule of skeletal muscle [11], 
Purkinje strands [9], crystalline lens of the frog eye [12], and syncytial tissues in general [2].

It is not true that any discretization of the original electroneutral model will exhibit stable solutions so long as the grid is coarse enough. The following finite difference scheme, in which the discrete solution values are defined at the edges of the voxels of the previous finite volume scheme, will be shown to be unstable when $L<0$ at all grid sizes.

Let $\tilde{p}_{k}(y, z, t)$ be an approximation of $\tilde{p}(k \Delta x, y, z, t)$ for $k=0,1,2, \ldots$ A finite difference approximation to the spatial derivative in $x$ gives a semidiscretized scheme for the diffusion equation:

$$
\frac{\partial \tilde{p}_{k}}{\partial t}=D\left(\frac{\partial^{2} \tilde{p}_{k}}{\partial y^{2}}+\frac{\partial^{2} \tilde{p}_{k}}{\partial z^{2}}+\frac{\tilde{p}_{k+1}-2 \tilde{p}_{k}+\tilde{p}_{k-1}}{(\Delta x)^{2}}\right),
$$

which holds for $k=1,2, \ldots$ A first-order discretization of the $x$-derivative in equation (3.15) gives

$$
L \frac{\partial \tilde{p}_{0}}{\partial t}=D\left(\frac{\tilde{p}_{1}-\tilde{p}_{0}}{\Delta x}\right) .
$$

Look for solutions of the form $\tilde{p}_{k}(y, z, t)=w^{k} \sin (\gamma y+\delta z) e^{\beta t}$ for $k=$ $1,2, \ldots$ With this ansatz, equations 3.29 and 3.30 become

$$
\begin{aligned}
& \frac{\beta}{D}=-\left(\gamma^{2}+\delta^{2}\right)+\frac{w-2+1 / w}{(\Delta x)^{2}}, \\
& \frac{\beta}{D}=\frac{w-1}{L \Delta x} .
\end{aligned}
$$

By equation (3.32), $\operatorname{Re}(\beta)$ has the opposite sign of $L$ since $|w|<1$ for a solution to decay in space, which implies $\operatorname{Re}(w-1)<0$. Thus, the semidiscretized scheme will be stable when $L>0$ and unstable when $L<0$ provided equations (3.31) and $(3.32$ ) have a solution with $|w|<1$. These solutions are

$$
w_{ \pm}=1+\frac{\chi+\chi^{2} \omega^{2}}{2(1+\chi)} \pm \frac{1}{2(1-\chi)} \sqrt{\left(\chi-\chi^{2} \omega^{2}\right)^{2}+4 \chi^{2} \omega^{2}}
$$

We find that $\left|w_{-}\right|<1$ for all $\chi \neq 1$ and $\omega>0$. Thus, there always exists a solution that decays in space. The stability of the finite difference scheme depends on the sign of $L$.

The same conclusion results when a second-order discretization of the $x$-derivative in equation (3.15) is made:

$$
L \frac{\partial \tilde{p}_{0}}{\partial t}=D\left(\frac{-\tilde{p}_{2}+4 \tilde{p}_{1}-3 \tilde{p}_{0}}{2 \Delta x}\right) .
$$

The same form for the solution gives

$$
\frac{\beta}{D}=\frac{(w-1)(3-w)}{2 L \Delta x} .
$$


Since $\operatorname{Re}((w-1)(3-w))<0$ when $|w|<1, \operatorname{Re}(\beta)$ has the opposite sign of $L$. Again, for all parameters there exists a solution satisfying $|w|<1$. A second-order discretization of the boundary condition did not change the fact that the stability of the semidiscrete equations depends on the sign of $L$. Furthermore, we have observed numerically that the stability of the finite difference discretization depends on the sign of $L$ also when the equations are fully discretized on a finite domain. This is not exhibited here.

\section{The Temporal Stabilization}

We shall now apply the temporal stabilization and demonstrate how temporal low-pass filtering in the space-charge layer boundary condition can stabilize the electroneutral model. Insert a lag into the boundary condition expressed in equation (3.15) by making $\lambda_{i}$ a dynamic variable that tracks, with strength $\alpha$, the value it would have in the original electroneutral model. Denote this target value, $\tilde{\lambda}_{i}$. The boundary condition becomes

$$
\begin{aligned}
\tilde{\lambda}_{i}(y, z, t) & =\frac{p_{i}(0, y, z, t)}{2 c_{*}}, \\
\frac{\partial \lambda_{i}}{\partial t}(y, z, t) & =\alpha\left(\tilde{\lambda}_{i}(y, z, t)-\lambda_{i}(y, z, t)\right), \\
D \frac{\partial p_{i}}{\partial x}(0, y, z, t) & =-\frac{C_{\mathrm{m}} \phi_{*}}{q} \frac{\partial \lambda_{i}}{\partial t}(y, z, t) .
\end{aligned}
$$

The parameter $\alpha>0$ controls the amount of temporal stabilization (with greater stabilization for smaller $\alpha$ ) and the original electroneutral model is realized in the limit as $\alpha \rightarrow \infty$.

Let $\tilde{p}=p_{1}-p_{2}$ and $\lambda=\lambda_{1}-\lambda_{2}$, to find that

$$
\begin{gathered}
\frac{\partial \tilde{p}}{\partial t}=D \Delta \tilde{p}, \\
\frac{\partial \tilde{p}}{\partial x}(\infty, y, z, t)=0, \\
\frac{\partial \tilde{p}}{\partial x}(0, y, z, t)=-\frac{C_{\mathrm{m}} \phi_{*}}{D q} \frac{\partial \lambda}{\partial t}(y, z, t), \\
\frac{\partial \lambda}{\partial t}(y, z, t)=\alpha[\tilde{\lambda}(y, z, t)-\lambda(y, z, t)], \\
\tilde{\lambda}(y, z, t)=\frac{\tilde{p}(0, y, z, t)}{2 c_{*}} .
\end{gathered}
$$

We seek a condition on $\alpha$ so that the problem for $\tilde{p}$ is well-posed.

Look for solutions of the form

$$
\begin{aligned}
& \tilde{p}(x, y, t)=e^{-\mu x} \sin (\gamma y+\delta z) e^{\beta t}, \\
& \lambda(y, z, t)=v \sin (\gamma y+\delta z) e^{\beta t} .
\end{aligned}
$$


It is important that $\tilde{p}$ and $\lambda$ have the same dependence on $y$ and $z$ as well as the same temporal growth/decay rate. We require that $\operatorname{Re}(\mu) \geq 0$ so that solutions are bounded in space. If $\operatorname{Re}(\mu)=0$, then equation (3.34) will be satisfied only after synthesis of the eigenfunctions. From this ansatz, we find that

$$
\begin{aligned}
& \frac{\beta}{D}+\gamma^{2}+\delta^{2}=\mu^{2}, \\
& -\mu D=-\frac{C_{\mathrm{m}} \phi_{*}}{q} \nu \beta, \\
& \nu \beta=\alpha\left(\frac{1}{2 c_{*}}-v\right) .
\end{aligned}
$$

From equation 3.42, $v$ is expressed in terms of $\beta$ as $v=\frac{\alpha}{\alpha+\beta} \frac{1}{2 c_{*}}$. Substituting this into equation (3.41) and solving for $\beta$ gives $\beta=-\frac{\mu \alpha D}{L \alpha+\mu D}$, where $L=$ $-\frac{C_{\mathrm{m}} \phi_{*}}{2 q c_{*}}$. This is the same expression for $L$ as in equation 3.15]. Substituting the expression for $\beta$ into equation 3.40 gives a cubic equation for $\mu$,

$$
\mu=\left(L+\frac{D}{\alpha} \mu\right)\left(\gamma^{2}+\delta^{2}-\mu^{2}\right) .
$$

For stability, we desire $\operatorname{Re}(\beta) \leq 0$ for all $\gamma>0$ and $\delta>0$. If $L>0$ then equation (3.43) has exactly one solution with $\operatorname{Re}(\mu)>0$ for all parameters that necessarily has $\operatorname{Re}(\beta)<0$. This stability agrees with that of the original electroneutral model.

When the original electroneutral model is unstable, $L<0$, the temporal stabilization is stable provided $\alpha$ is not too large. Equation (3.43) has one solution with $\operatorname{Re}(\mu)<0$ and two solutions with $\operatorname{Re}(\mu)>0$ for all parameters. The two solutions with $\operatorname{Re}(\mu)>0$ correspond to $\operatorname{Re}(\beta)<0$ depending on two dimensionless parameter combinations: a dimensionless stabilization strength, $\kappa=\frac{\alpha L^{2}}{D}$, and a dimensionless frequency, $\omega=|L| \sqrt{\gamma^{2}+\delta^{2}}$. This dependence is shown in Figure 3.3 . The regions shaded darkest to lightest, have the properties that both solutions are real with $\beta<0$, both solutions are complex conjugates with $\operatorname{Re}(\beta)<0$, both solutions are complex conjugates with $\operatorname{Re}(\beta)>0$, and both solutions are real with $\beta>0$ respectively. By considering the discriminant of the cubic, equation (3.43), we find that the regions in which the solutions are real are separated from the regions in which the solutions are complex by a curve given implicitly by

$$
\kappa^{4}-4 \kappa^{3}-20 \kappa^{3} \omega^{2}-8 \kappa^{2} \omega^{4}+4 \kappa^{4} \omega^{2}+12 \kappa^{2} \omega^{2}-12 \kappa \omega^{4}+4 \omega^{6}=0 .
$$

An equation for the curve that separates the two regions with complex conjugate solutions and different signs of $\operatorname{Re}(\beta)$ is obtained by setting $\operatorname{Re}(\beta)=0$ and eliminating $\operatorname{Im}(\beta)$ and $\mu$ from equation (3.40) and equation 3.43). The equation for the curve is 


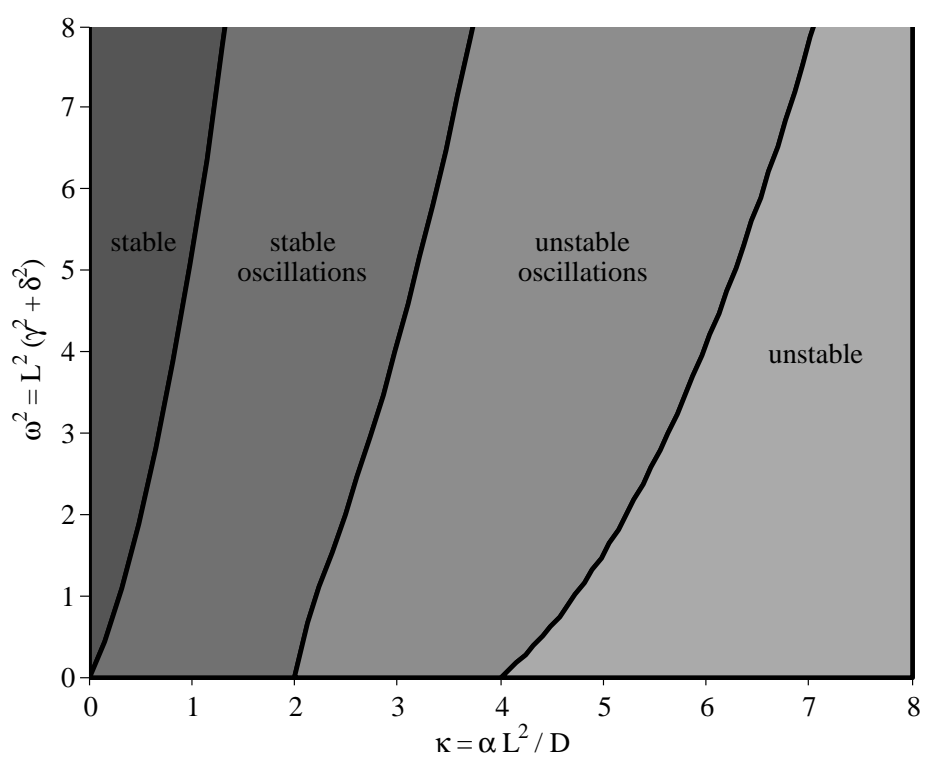

FIGURE 3.3. Properties of the two solutions of equation (3.43) with $\operatorname{Re}(\mu)>0$ for $L<0$. The four regions shown, from darkest to lightest, have the following properties: both solutions are real with $\beta<0$, both solutions are complex conjugates with $\operatorname{Re}(\beta)<0$, both solutions are complex conjugates with $\operatorname{Re}(\beta)>0$, and both solutions are real with $\beta>0$. Parameters from the first two regions result in stable solutions of the form presented in equations (3.44) and 3.45. The only values of $\kappa$ for which the solutions will be stable for all $\omega$ are $0<\kappa<2$.

$$
\omega^{2}=\frac{\kappa^{2}-2 \kappa}{2}+\frac{\kappa}{2} \sqrt{\kappa^{2}-2 \kappa}
$$

The salient feature of this curve is that when $\omega^{2}=0, \kappa=2$. Thus, $\operatorname{Re}(\beta)$ will be negative for all $\gamma$ and $\delta$ precisely when $\kappa<2$ or

$$
\frac{1}{\alpha}>\frac{L^{2}}{2 D}
$$

Stability occurs when the low-pass filtering of the boundary condition attenuates the high temporal frequencies.

We shall now show that the solutions to the temporal stabilization of the electroneutral model agree with those of the PNP model transiently. We consider the oscillatory solutions explicitly by seeking solutions of the form

$$
\begin{aligned}
& \tilde{p}(x, y, t)=\cos (\mu x+\theta) \sin (\gamma y+\delta z) e^{\beta t}, \\
& \lambda(y, z, t)=v \sin (\gamma y+\delta z) e^{\beta t} .
\end{aligned}
$$


with $\mu$ real and positive. We find that

$$
\frac{\beta}{D}+\gamma^{2}+\delta^{2}=-\mu^{2}
$$

These solutions are stable for all $\gamma$ and $\delta$. Additionally, $\mu>0$ satisifies

$$
-\left(\mu^{2}+\gamma^{2}+\delta^{2}\right)(L \alpha+\mu D \tan \theta)=-\mu \alpha \tan \theta .
$$

For each $\mu>0$, there is a $\theta$ that satisfies equation (3.47). These stable eigenfunctions approach those of the PNP model as $c_{*} \rightarrow \infty$. We take $l_{\mathrm{D}} \rightarrow 0, \theta \rightarrow 0$, and $\alpha \rightarrow \infty$ so that $L \alpha$ and $\tan \theta / L$ are fixed. In this limit, we find that two of the solutions for $\mu$ of equation (3.47) approach those given in equation (3.20) and the third rushes off to infinity. The eigenfunctions corresponding to the values of $\mu$ from equation (3.20) have previously been shown to be in agreement with those of the PNP model in the limit as $c_{*} \rightarrow \infty$. The limiting decay rate, $\beta$, from the two models agree since equations (3.46) and (3.16) with $\eta=\mu^{2}$ are the same.

The eigenfunctions given in equation (3.38) with $\mu$ given by equation (3.43) approach constant-in- $x$ solutions as $c_{*} \rightarrow \infty$. We take $l_{\mathrm{D}} \rightarrow 0$ and $\alpha \rightarrow \infty$ so that $L \alpha$ is fixed. In this limit, two of the roots of equation 3.43 become complex and rush off to infinity. The remaining root goes to 0 . The solution with $\mu \rightarrow 0$ is stable since $\frac{L^{2} \alpha}{2 D} \rightarrow 0$ and the condition in equation $(3.2)$ is satisfied. The limiting decay rate, $\beta$, from the two models agree since equations (3.40) and 3.16) with $\eta=-\mu^{2}$ are the same.

\section{Conclusion}

We have outlined the electroneutral model and its derivation from the PNP model of electrodiffusion. Through a particular transient electrodiffusion problem, we have confirmed, as observed in [13], that the original electroneutral model is ill-posed. We have introduced a modification to the electroneutral model, the finite thickness stabilization. A previous modification, the temporal stabilization, and stabilization by a finite volume discretization were also considered. These stabilizations were shown to be well-posed for the example problem and good approximations to the PNP model.

Future work includes proving that the modified electroneutral models are wellposed for all electrodiffusion problems including those with permeable membranes, multivalent ions, more than two ion types, and unequal diffusion coefficients. The example herein presented was selected so that the electrostatic potential could be obtained independently of the ion concentrations. This removes the nonlinearity from the problem and makes it amenable to an eigenfunction decomposition. We believe that the example considered here is illustrative of more general electrodiffusion problems despite its limitations.

In general, understanding the role of ionic solutions in physiology is important. We hope that the analysis and discussion presented here will be helpful in the practical application of the electroneutral model to electrodiffusion problems. 
Specifically, we point out three biological systems previously studied that could benefit from the electroneutral model: electrodiffusion in the node of Ranvier [10], horizontal cells of the retina [5], and the local field potential near a cylindrical axon with Hodgkin-Huxley transmembrane currents [17]. Careful consideration of the analysis of the space-charge layer and the other underlying assumptions of the electroneuronal model may permit the development of tractable models for a broader range of complex electrodiffusion phenomena. The problems of access to a channel [8] and the interplay between the intricate calcium channel and calcium domains [1] are potential targets.

Acknowledgments. The authors would like to acknowledge helpful conversations with Bob Eisenberg and Percy Deift. ARS was supported by National Science Foundation Grant DMS-1009575 and a fellowship from the Graduate School of Arts and Sciences at New York University.

\section{Bibliography}

[1] Chad, J. E.; Eckert, R. Calcium domains associated with individual channels can account for anomalous voltage relations of Ca-dependent responses. Biophys. J. 45 (1984), no. 5, 993-999. doi:10.1016/S0006-3495(84)84244-7

[2] Eisenberg, R. S.; Barcilon, V.; Mathias, R. T. Electrical properties of spherical syncytia. Biophys. J. 25 (1979), no. 1, 151-180. doi:10.1016/S0006-3495(79)85283-2

[3] Everitt, C. T.; Haydon, D. A. Electrical capacitance of a lipid membrane separating two aqueous phases. J. Theor. Biol. 18 (1968), no. 3, 371-379. doi:10.1016/0022-5193(68)90084-2

[4] Fogolari, F.; Brigo, A.; Molinari, H. The Poisson-Boltzmann equation for biomolecular electrostatics: a tool for structural biology. J. Mol. Recogn. 15 (2002), no. 6, 377-392. doi:10.1002/jmr.577

[5] Gardner, C. L.; Jones, J. R.; Baer, S. M.; Chang, S. Simulation of the ephaptic effect in the cone-horizontal cell synapse of the retina. SIAM J. Appl. Math. 73 (2013), no. 2, 636-648. doi:10.1137/120878409

[6] Hyon, Y.; Eisenberg, B.; Liu, C. A mathematical model for the hard sphere repulsion in ionic solutions. Commun. Math. Sci. 9 (2011), no. 2, 459-475.

[7] Kragh, H. Dirac: a scientific biography. Cambridge University Press, Cambridge, 1990.

[8] Levadny, V.; Aguilella, V. M.; Belaya, M. Access resistance of a single conducting membrane channel. BBA-Biomembranes 1368 (1998), no. 2, 338-342. doi:10.1016/S00052736(97)00205-8

[9] Levis, R. A.; Mathias, R. T.; Eisenberg, R. S. Electrical properties of sheep Purkinje strands. Electrical and chemical potentials in the clefts. Biophys. J. 44 (1983), no. 2, 225-248. doi:10.1016/S0006-3495(83)84295-7

[10] Lopreore, C. L.; Bartol, T. M.; Coggan, J. S.; Keller, D. X.; Sosinsky, G. E.; Ellisman, M. H.; Sejnowski, T. J. Computational modeling of three-dimensional electrodiffusion in biological systems: application to the node of Ranvier. Biophys. J. 95 (2008), no. 6, 2624-2635. doi:10.1529/biophysj.108.132167

[11] Mathias, R. T.; Eisenberg, R. S.; Valdiosera, R. Electrical properties of frog skeletal muscle fibers interpreted with a mesh model of the tubular system. Biophys. J. 17 (1977), no. 1, 57-93. doi:10.1016/S0006-3495(77)85627-0

[12] Mathias, R. T.; Rae, J. L.; Eisenberg, R. S. Electrical properties of structural components of the crystalline lens. Biophys. J. 25 (1979), no. 1, 181-201. doi:10.1016/S0006-3495(79)85284-4 
[13] Mori, Y. A three-dimensional model of cellular electrical activity. Doctoral dissertation, Courant Institute of Mathematical Sciences, 2006.

[14] Mori, Y.; Fishman, G. I.; Peskin, C. S. Ephaptic conduction in a cardiac strand model with 3D electrodiffusion. Proc. Natl. Acad. Sci. USA 105 (2008), no. 17, 6463-6468. doi:10.1073/pnas.0801089105

[15] Mori, Y.; Jerome, J. W.; Peskin, C. S. A three-dimensional model of cellular electrical activity. Bull. Inst. Math. Acad. Sin. (N.S.) 2 (2007), no. 2, 367-390.

[16] Mori, Y.; Peskin, C. S. A numerical method for cellular electrophysiology based on the electrodiffusion equations with internal boundary conditions at membranes. Commun. Appl. Math. Comput. Sci. 4 (2009), 85-134. doi:10.2140/camcos.2009.4.85

[17] Pods, J.; Schönke, J.; Bastian, P. Electrodiffusion models of neurons and extracellular space using the Poisson-Nernst-Planck equations - numerical simulation of the intraand extracellular potential for an axon model. Biophys. J. 105 (2013), no. 1, 242-254. doi:10.1016/j.bpj.2013.05.041

[18] Rubinstein, I. Electro-diffusion of ions. SIAM Studies in Applied Mathematics, 11. Society for Industrial and Applied Mathematics (SIAM), Philadelphia, 1990. doi:10.1137/1.9781611970814

[19] White, S. H. A study of lipid bilayer membrane stability using precise measurements of specific capacitance. Biophys. J. 10 (1970), no. 12, 1127-1148. doi:10.1016/S0006-3495(70)86360-3

\section{AdAM R. STINCHCOMBE \\ Department of Mathematics \\ University of Michigan \\ 530 Church St. \\ Ann Arbor, MI 48109 \\ USA}

E-mail: stincheumich.edu

\section{YOICHIRO MORI}

School of Mathematics

University of Minnesota

206 Church St.

Minneapolis, MN 55455

USA

E-mail:ymori@umn.edu

Charles S. Peskin

Courant Institute

New York University

251 Mercer St.

New York, NY 10012

USA

E-mail: peskinecims.nyu.edu

Received December 2014. 